Research Article

\title{
Estimates for the Finite-Time Ruin Probability of a Time-Dependent Risk Model with a Brownian Perturbation
}

\author{
Kaiyong Wang ${ }^{(D}$, Yongfang Cui, and Yanzhu Mao \\ School of Mathematics and Physics, Suzhou University of Science and Technology, Suzhou 215009, China \\ Correspondence should be addressed to Kaiyong Wang; beewky@vip.163.com
}

Received 10 January 2020; Accepted 30 January 2020; Published 18 February 2020

Guest Editor: Wenguang Yu

Copyright ( $\odot 2020$ Kaiyong Wang et al. This is an open access article distributed under the Creative Commons Attribution License, which permits unrestricted use, distribution, and reproduction in any medium, provided the original work is properly cited.

In this paper, we consider a time-dependent risk model with a Brownian perturbation. In this model, there is a dependence structure between the claim sizes and their corresponding interarrival times. Assuming the claim sizes have subexponential distributions, we obtain the asymptotic lower bound of the finite-time ruin probability. When the claim sizes have distributions from the class $L \cap D$, the asymptotic upper bound of the finite-time ruin probability has been presented. These results confirm that when the claim sizes are heavy-tailed, the asymptotics of the finite-time ruin probability of this time-dependent model are insensitive to the Brownian perturbation.

\section{Introduction}

This paper considers a dependent risk model with a constant force of interest and Brownian perturbation. In such a model, the claim sizes form a sequence of independent and identically distributed (i.i.d.) nonnegative random variables $X_{n}, n \geq 1$, with a distribution $F$ and the interarrival times are also a sequence of i.i.d. nonnegative random variables $\theta_{n}, n \geq 1$, with a distribution $G$. In this paper, we consider there exists a dependence structure between the claim sizes $X_{n}, n \geq 1$, and their corresponding interarrival times $\theta_{n}, n \geq 1$. The claim arrival times are denoted by $\tau_{n}=\sum_{i=1}^{n} \theta_{i}, n \geq 1$. The renewal counting process

$$
N(t)=\sup \left\{n \geq 1: \tau_{n} \leq t\right\}, \quad t \geq 0,
$$

is the number of claims by time $t$. Suppose that $N(t), t \geq 0$, has a finite mean:

$$
\lambda(t)=\mathrm{EN}(t)=\sum_{n=1}^{\infty} P\left(\tau_{n} \leq t\right), \quad t \geq 0 .
$$

Then, the amount of aggregate claim sizes is $S(t)=$ $\sum_{n=1}^{N(t)} X_{n}, t \geq 0$, where by convention, $\sum_{n=1}^{0}=0$. Suppose that, in this risk model, there is a constant force of interest $r \geq 0$. The discounted aggregate claim size by time $t$ is

$$
D(t)=\int_{0^{-}}^{t} e^{-\mathrm{ru}} S(\mathrm{du})=\sum_{n=1}^{\infty} X_{n} e^{-r \tau_{n}} 1_{\left\{\tau_{n} \leq t\right\}}, \quad t \geq 0,
$$

where $1_{A}$ is the indicator function of an event $A$. Then, the insurer's surplus process of an insurance company by time $t$ is

$$
\begin{array}{r}
U(t)=e^{\mathrm{rt}} x+\int_{0}^{t} e^{r(t-s)} C(\mathrm{~d} s)-e^{\mathrm{rt}} D(t)+\delta \int_{0}^{t} e^{r(t-s)} B(\mathrm{~d} s), \\
t \geq 0,
\end{array}
$$

where $x \geq 0$ is the initial surplus; $C(t)$ is the premium income up to time $t, t \geq 0$, which is a nondecreasing and nonnegative stochastic process satisfying $C(0)=0$ and $C(t)<\infty, 0<t<\infty$, almost surely; $B(t), t \geq 0$, is a diffusion perturbation which is a standard Brownian motion, and $\delta \geq 0$ is a volatility factor. Throughout the paper, we suppose that $\{C(t), t \geq 0\},\left\{\left(X_{n}, \theta_{n}\right), n \geq 1\right\}$, and $\{B(t), t \geq 0\}$ are mutually independent. For every $0<t<\infty$, let $\widetilde{C}(t)=\int_{0}^{t} e^{-\mathrm{rs}} C(\mathrm{~d} s)$. The finite-time ruin probability up to time $t$ is defined as

$$
\psi(x, t)=P\left(\inf _{0<s \leq t} U(s)<0 \mid U(0)=x\right), \quad t \geq 0 .
$$


This paper considers the asymptotics for $\psi(x, t)$ as $x$ tends to $\infty$ under the heavy-tailed claim sizes.

When the claim sizes $X_{n}, n \geq 1$, and the interarrival time $\theta_{n}, n \geq 1$, are independent, there are many literatures to investigate ruin probabilities for claim sizes with independent/dependent structure (see, e.g., Asmussen [1], Chen and $\mathrm{Ng}$ [2], Hao and Tang [3], Kalashnikov and Konstantinides [4], Klüppelberg and Stadtmüller [5], Konstantinides et al. [6], Wang et al. [7], Dong et al. [8], Fu et al. [9], Li [10], Peng et al. [11], Peng and Wang [12, 13], Yang et al. [14], Yu et al. [15], and Zhang et al. [16]). However, in the last few years, many researchers have proposed some dependent risk models with various dependence structures modeling the dependence between the claim sizes and their corresponding interarrival times (see, e.g., Albrecher and Teugels [17], Boudreault et al. [18], Cossette et al. [19], Badescu et al. [20], and Asmussen et al. [21]).

In this paper, we use the dependence structure proposed by Asimit and Badescu [22]. Assume that $\left(X_{n}, \theta_{n}\right), n \geq 1$, are i.i.d. copies of a generic pair $(X, \theta)$ with dependent components $X$ and $\theta$ satisfying

$$
P(X>x \mid \theta=t) \sim \bar{F}(x) h(t),
$$

holds uniformly for $t \geq 0$, where $h(\cdot)$ : $[0, \infty)$ is a measurable function and the symbol $\sim$ means that the quotient of both sides tends to 1 as $x \longrightarrow \infty$. Asimit and Badescu [22] and Li et al. [23] have shown that (6) can be satisfied by many copulas, such as Ali-Mikhail-Haq copula, Farlie-Gumbel-Morgenstern copula, and Frank copula.

Using the uniformity of (6), we know that $\operatorname{Eh}(\theta)=1$. Thus, we can define a random variable $\theta_{1}^{*}$, which is independent of $\left\{X_{n}, n \geq 1\right\}, \quad\left\{\theta_{n}, n \geq 1\right\}, \quad\{C(t), t \geq 0\}$, and $\{B(t), t \geq 0\}$ and has the following distribution:

$$
G^{*}(\mathrm{~d} t)=h(t) G(\mathrm{~d} t), \quad t \geq 0
$$

Define $\tau_{1}^{*}=\theta_{1}^{*}$ and $\tau_{n}^{*}=\theta_{1}^{*}+\sum_{i=2}^{n} \theta_{i}, n \geq 2$, which constitute a delayed renewal counting process

$$
N^{*}(t)=\sup \left\{n \geq 1: \tau_{n}^{*} \leq t\right\}, \quad t \geq 0,
$$

with a mean function

$$
\tilde{\lambda}(t)=\mathrm{EN}^{*}(t)=\int_{0^{-}}^{t}(1+\lambda(t-u)) h(u) G(\mathrm{~d} u), \quad t \geq 0 .
$$

For the risk model (4), when the claim sizes $X_{n}, n \geq 1$, and the interarrival times $\theta_{n}, n \geq 1$, are independent and the premium income process $C(t), t \geq 0$, is a linear process, $\mathrm{Li}$ [10] provided the asymptotics of the finite-time ruin probability for subexponential claim sizes. Wang et al. [24] considered a risk model with stochastic return. This paper considers there exists a dependence structure (6) between the claim sizes $X_{n}, n \geq 1$, and their corresponding interarrival times $\theta_{n}, n \geq 1$, and provides the estimates of the finite-time ruin probability of this model with heavy-tailed claim sizes.

In this paper, unless stated otherwise, all limits are for $x \longrightarrow \infty$. For two positive functions $u(\cdot)$ and $v(\cdot)$, if $\limsup u(x) / v(x) \leq 1$, then we write $u(x) \lesssim v(x)$; if $\lim \inf u(x) / v(x) \geq 1$, then we write $u(x) \geq v(x)$; if $\lim u(x) / v(x)=0$, then we write $u(x)=o(v(x))$; if $\limsup u(x) / v(x)<\infty$, then we write $u(x)=O(v(x))$. For a distribution $V$ on $(-\infty, \infty)$, let $\bar{V}(x)=1-V(x), x \in$ $(-\infty, \infty)$, be its (right) tail.

For a distribution $V$ on $[0, \infty)$, if $\int_{0}^{\infty} e^{\lambda y} V(\mathrm{~d} y)=\infty$ for any $\lambda>0$, then we say that $V$ is heavy-tailed; otherwise, we say that $V$ is light-tailed. In the following, we introduce some subclasses of heavy-tailed distribution class. Say that a distribution $V$ on $[0, \infty)$ belongs to the subexponential distribution class $S$, if

$$
\overline{V^{* n}}(x) \sim n \bar{V}(x),
$$

holds for some (or equivalently for all) $n \geq 2$, where $V^{* n}$ is the $n$-fold convolution of $V$. Lemma 1.3.5(b) of Embrechts et al. [25] shows that if $V \in \mathscr{P}$, then for any $\varepsilon>0$,

$$
e^{-\varepsilon x}=o(\bar{V}(x))
$$

Say that a distribution $V$ on $[0, \infty)$ belongs to the longtailed distribution class $L$, if for any $y>0$,

$$
\bar{V}(x+y) \sim \bar{V}(x) \text {. }
$$

By the uniform convergence theorem for slowly varying functions (Theorem A3.2 of Embrechts et al. [25]), this convergence also holds uniformly for every compact set of $y$. Say that a distribution $V$ on $[0, \infty)$ belongs to the dominated varying distribution class $D$, if for any $0<y<1$,

$$
\bar{V}(x y)=O(\bar{V}(x)) \text {. }
$$

The above distribution classes have the following proper relation:

$$
L \cap D \subset S \subset L,
$$

(see, e.g., Embrechts et al. [25] and Klüppelberg and Stadtmüller [5]). The following is the main result of this paper.

Theorem 1. Consider the risk model (4). Assume that $\left(X_{n}, \theta_{n}\right), n \geq 1$, are i.i.d. and satisfy (6).

(1) If $F \in \mathcal{S}$, then for each $t>0$,

$$
\psi(x, t) \gtrsim \int_{0^{-}}^{t} \bar{F}\left(\mathrm{xe}^{\mathrm{rs}}\right) \tilde{\lambda}(\mathrm{d} s) .
$$

(2) If $F \in \mathscr{L} \cap \mathscr{D}$, then for each $t>0$,

$$
\psi(x, t) \lesssim \int_{0^{-}}^{t} \bar{F}\left(\mathrm{xe}^{\mathrm{rs}}\right) \widetilde{\lambda}(\mathrm{d} s) .
$$

Because $L \cap D \subset S$, from Theorem 1 , we can obtain the following corollary.

Corollary 1. Consider the risk model (4). Assume that $\left(X_{n}, \theta_{n}\right), n \geq 1$, are i.i.d. and satisfy (6). If $F \in \mathscr{L} \cap \mathscr{D}$, then for each $t>0$,

$$
\psi(x, t) \sim \int_{0^{-}}^{t} \bar{F}\left(\mathrm{xe}^{\mathrm{r} s}\right) \tilde{\lambda}(\mathrm{d} s)
$$


Remark 1. When the claim sizes $X_{n}, n \geq 1$, and the interarrival times $\theta_{n}, n \geq 1$, are independent, Li [10] considered the risk model (4) for a linear premium income process $C(t), t \geq 0$, and subexponential claim sizes. He obtained

$$
\psi(x, t) \sim \int_{0^{-}}^{t} \bar{F}\left(\mathrm{xe}^{\mathrm{rs}}\right) \lambda(\mathrm{d} s),
$$

holds uniformly for $t$ in a finite-time interval. This result indicates that when the claim sizes are heavy-tailed, the asymptotics of the finite-time ruin probability is insensitive to a Brownian perturbation. Theorem 1 confirms that when there exists the dependence structure (6) between the claim sizes $X_{n}, n \geq 1$, and their corresponding interarrival times $\theta_{n}, n \geq 1$, the asymptotics of the finite-time ruin probability is also insensitive to a Brownian perturbation for heavy-tailed claim sizes.

\section{Proof of the Main Result}

Lemma 1. Consider the risk model (4). Assume that $\left(X_{n}, \theta_{n}\right)$, $n \geq 1$, are i.i.d. and satisfy (6).

(1) If $F \in S$, then for each $t>0$,

$$
P(D(t)>x) \sim \int_{0^{-}}^{t} \bar{F}\left(\mathrm{xe}^{\mathrm{rs}}\right) \tilde{\lambda}(\mathrm{d} s) .
$$

(2) If $F \in L \cap D$, then for each $t>0$, the distribution of $D(t)$ belongs to the class $L \cap D$.

Proof. The result (1) is from Theorem 2.1 of Li et al. [23]. Now, we prove (2). Because $L \cap D \subset S$, for each $t>0$, relation (19) holds.

For every $y>0$ and any $0 \leq s \leq t, y \leq \mathrm{ye}^{\mathrm{rs}} \leq \mathrm{ye}^{\mathrm{rt}}$. Because $F \in L$, by the local uniformity of the class $\mathscr{L}$, it holds uniformly for $0 \leq s \leq t$ that

$$
\bar{F}\left(x+\mathrm{ye}^{\mathrm{rs}}\right) \sim \bar{F}(x) .
$$

This means that, for any $\varepsilon>0$, there exists $x_{0}=x_{0}(\varepsilon, y, t)>0$ such that for any $x>x_{0}$ and $0 \leq s \leq t$,

$$
(1-\varepsilon) \bar{F}(x) \leq \bar{F}\left(x+\mathrm{ye}^{\mathrm{rs}}\right) \leq(1+\varepsilon) \bar{F}(x) .
$$

Hence, for any $x>x_{0}$ and $0 \leq s \leq t$,

$$
(1-\varepsilon) \bar{F}\left(\mathrm{xe}^{\mathrm{rs}}\right) \leq \bar{F}\left((x+y) e^{\mathrm{rs}}\right) \leq(1+\varepsilon) \bar{F}\left(\mathrm{xe}^{\mathrm{rs}}\right) .
$$

By (22), it holds that

$$
\begin{aligned}
(1-\varepsilon) \int_{0^{-}}^{t} \bar{F}\left(\mathrm{xe}^{\mathrm{rs}}\right) \tilde{\lambda}(\mathrm{d} s) & \leq \int_{0^{-}}^{t} \bar{F}\left((x+y) e^{\mathrm{rs}}\right) \tilde{\lambda}(\mathrm{d} s) \\
& \leq(1+\varepsilon) \int_{0^{-}}^{t} \bar{F}\left(\mathrm{xe}^{\mathrm{r} s}\right) \tilde{\lambda}(\mathrm{d} s)
\end{aligned}
$$

that is,

$$
\int_{0^{-}}^{t} \bar{F}\left((x+y) e^{\mathrm{rs}}\right) \tilde{\lambda}(\mathrm{d} s) \sim \int_{0^{-}}^{t} \bar{F}\left(\mathrm{xe}^{\mathrm{r} s}\right) \widetilde{\lambda}(\mathrm{d} s) .
$$

By (19) and (24), we know that the distribution of $D(t)$ belongs to the class $L$. Next, we will prove the distribution of $D(t)$ belongs to the class $D$. Because $F \in D$, for every $0<y<1$, there exist constants $M>0$ and $x_{1}=x_{1}(y)>0$ such that for any $x>x_{1}$ and $0 \leq s \leq t$,

$$
\bar{F}\left(\mathrm{xye}^{\mathrm{rs}}\right) \leq M \bar{F}\left(\mathrm{xe}^{\mathrm{rs}}\right) \text {. }
$$

Here, for any $x>x_{1}$,

$$
\int_{0^{-}}^{t} \bar{F}\left(\mathrm{xye}^{\mathrm{rs}}\right) \tilde{\lambda}(\mathrm{d} s) \leq M \int_{0^{-}}^{t} \bar{F}\left(\mathrm{xe}^{\mathrm{r} s}\right) \tilde{\lambda}(\mathrm{d} s) .
$$

By (19) and (26), for every $0<y<1$, it holds that

$$
\limsup _{x \rightarrow \infty} \frac{P(D(t)>x y)}{P(D(t)>x)} \leq M
$$

that is, the distribution of $D(t)$ belongs to the class $D$.

The next lemma is Lemma 4.2 of Li et al. [23].

Lemma 2. Consider the risk model (4). Assume that $\left(X_{n}, \theta_{n}\right)$, $n \geq 1$, are i.i.d. and satisfy (6). If $F \in S$, then for every $n \geq 1$, it holds for each $t>0$ that

$$
P\left(\sum_{i=1}^{n} X_{i} e^{-r \tau_{i}}>x, N(t)=n\right) \sim \sum_{i=1}^{n} P\left(X_{i} e^{-r \tau_{i}}>x, N(t)=n\right) .
$$

The following lemma slightly extends the above lemma.

Lemma 3. Consider the risk model (4). Assume that $\left(X_{n}, \theta_{n}\right)$, $n \geq 1$, are i.i.d. and satisfy (6). Suppose that $\xi$ is a nonnegative random variable and independent of other random variables. If $F \in S$, then for every $n \geq 1$ and each $t>0$, it holds that

$$
P\left(\sum_{i=1}^{n} X_{i} e^{-r \tau_{i}}>x+\xi, N(t)=n\right) \sim \sum_{i=1}^{n} P\left(X_{i} e^{-r \tau_{i}}>x, N(t)=n\right) .
$$

Proof. Because $\xi$ is nonnegative, by Lemma 2, for every $n \geq 1$ and each $t>0$,

$$
\begin{aligned}
& P(\left.\sum_{i=1}^{n} X_{i} e^{-r \tau_{i}}>x+\xi, N(t)=n\right) \leq P\left(\sum_{i=1}^{n} X_{i} e^{-r \tau_{i}}>x, N(t)=n\right) \\
& \sim \sum_{i=1}^{n} P\left(X_{i} e^{-r \tau_{i}}>x, N(t)=n\right) .
\end{aligned}
$$

Next, we prove that, for every $n \geq 1$ and each $t>0$,

$$
P\left(\sum_{i=1}^{n} X_{i} e^{-r \tau_{i}}>x+\xi, N(t)=n\right) \gtrless \sum_{i=1}^{n} P\left(X_{i} e^{-r \tau_{i}}>x, N(t)=n\right) .
$$

Because $\xi$ is nonnegative and independent of other random variables, by Lemma 2 , for every $n \geq 1$ and each $t>0$,

$$
\begin{aligned}
P( & \left.\sum_{i=1}^{n} X_{i} e^{-r \tau_{i}}>x+\xi, N(t)=n\right) \\
& \sim \sum_{i=1}^{n} P\left(X_{i} e^{-r \tau_{i}}>x+\xi, N(t)=n\right) .
\end{aligned}
$$


Because $F \in S \subset L$, by the dominated convergence theorem, for each $t>0$, it holds uniformly for $1 \leq i \leq n$ that

$$
\lim _{x \longrightarrow \infty} \frac{P\left(X_{i}>x+e^{\mathrm{rt}} \xi\right)}{P\left(X_{i}>x\right)}=\int_{0^{-}}^{\infty} \lim _{x \longrightarrow \infty} \frac{\bar{F}\left(x+\mathrm{ye}^{\mathrm{rt}}\right)}{\bar{F}(x)} P(\xi \in \mathrm{d} y)=1 .
$$

Hence, for any $\varepsilon>0$, there exists a constant $x_{3}=x_{3}(\varepsilon, t, n)>0$ such that for any $x>x_{3}$ and $1 \leq i \leq n$,

$$
P\left(X_{i}>x+e^{\mathrm{rt}} \xi\right) \geq(1-\varepsilon) P\left(X_{i}>x\right) .
$$

For every $t>0$ and $n \geq 1$, set $\Omega_{n}(t)=\left\{\left(s_{1}, \ldots, s_{n}\right) \in\right.$ $\left.[0, t]^{n}: t_{n}=\sum_{i=1}^{n} s_{i} \leq t\right\}$. By (6), (32), and (34), for every $n \geq 1$, each $t>0$, and $x>x_{3}$, it holds that

$$
\begin{aligned}
& P\left(\sum_{i=1}^{n} X_{i} e^{-r \tau_{i}}>x+\xi, N(t)=n\right) \\
& \quad \sim \sum_{i=1}^{n} P\left(X_{i} e^{-r \tau_{i}}>x+\xi, N(t)=n\right) \\
& \quad=\sum_{i=1}^{n} P\left(X_{i} e^{-r \tau_{i}}>x+\xi, \tau_{n} \leq t, \tau_{n+1}>t\right) \\
& =\sum_{i=1}^{n} \int_{\Omega_{n}(t)} \int P\left(X_{i} e^{-r \mathrm{rt}_{i}}>x+\xi \mid \theta_{i}=s_{i}\right) \bar{G}\left(t-t_{n}\right) \prod_{i=1}^{n} G\left(\mathrm{~d} s_{i}\right) \\
& \quad \sim \sum_{i=1}^{n} \int_{\Omega_{n}(t)} \int P\left(X_{i} e^{-r \mathrm{rt}_{i}}>x+\xi\right) h\left(s_{i}\right) \bar{G}\left(t-t_{n}\right) \prod_{i=1}^{n} G\left(\mathrm{~d} s_{i}\right) \\
& \quad \geq \sum_{i=1}^{n} \int_{\Omega_{n}(t)} \int P\left(X_{i}>\mathrm{xe}^{\mathrm{rt}}+e^{\mathrm{rt}} \xi\right) h\left(s_{i}\right) \bar{G}\left(t-t_{n}\right) \prod_{i=1}^{n} G\left(\mathrm{~d} s_{i}\right) \\
& \quad \geq(1-\varepsilon) \sum_{i=1}^{n} \int_{\Omega_{n}(t)} \int P\left(X_{i}>\mathrm{xe}^{\mathrm{rt}}\right) h\left(s_{i}\right) \bar{G}\left(t-t_{n}\right) \prod_{i=1}^{n} G\left(\mathrm{~d} s_{i}\right) \\
& \quad \sim(1-\varepsilon) \sum_{i=1}^{n} P\left(X_{i} e^{-r \tau_{i}}>x, N(t)=n\right),
\end{aligned}
$$

that is, (31) holds.

The following lemma is derived from Proposition 1(a) of Embrechts et al. [26].

Lemma 4. Let $V_{i}, i=1,2$ be two distributions on $[0, \infty)$. If $V_{1} \in \mathcal{S}$ and $\overline{V_{2}}(x)=o\left(\overline{V_{1}}(x)\right)$, then

$$
\overline{V_{1} * V_{2}}(x) \sim \overline{V_{1}}(x) \text {. }
$$

For the above risk model, write

$$
p(t)=\delta \int_{0}^{t} e^{-\mathrm{rs}} B(\mathrm{~d} s), \quad t \geq 0 .
$$

Let

$$
\begin{aligned}
& p_{*}(t)=\inf _{0 \leq s \leq t} p(s) \leq 0, \quad t \geq 0, \\
& p^{*}(t)=\sup _{0 \leq s \leq t} p(s) \geq 0, \quad t \geq 0 .
\end{aligned}
$$

The following lemma can be obtained from Theorem D.3 (ii) of Piterbarg [27].
Lemma 5. For each $t>0$,

$$
P\left(p_{*}(t)<-x\right)=P\left(p^{*}(t)>x\right) \sim 2 \bar{\Phi}\left(\frac{\sqrt{2 r}}{\delta \sqrt{1-e^{-2 \mathrm{rt}}}} x\right),
$$

where $\bar{\Phi}(x) \sim 1 / \sqrt{2 \pi} x e^{-x^{2} / 2}$ is the tail of a standard normal distribution, and when $r=0, \sqrt{2 r} / \sqrt{1-e^{-2 r t}}$ is $1 / \sqrt{t}$ (i.e., a limit as $r \longrightarrow 0$ ).

Proof of Theorem 1. By Lemma 3, for each $t>0$ and any integer $m>0$, it holds that

$$
\begin{aligned}
\psi(x, t) \geq & P\left(\sum_{i=1}^{N(t)} X_{i} e^{-r \tau_{i}}-\widetilde{C}(t)-p^{*}(t)>x\right) \\
\geq & \sum_{n=1}^{m} P\left(\sum_{i=1}^{n} X_{i} e^{-r \tau_{i}}>x+\widetilde{C}(t)+p^{*}(t), N(t)=n\right) \\
& \sim \sum_{n=1}^{m} \sum_{i=1}^{n} P\left(X_{i} e^{-r \tau_{i}}>x, N(t)=n\right) \\
= & \left(\sum_{n=1}^{\infty}-\sum_{n=m+1}^{\infty}\right) \sum_{i=1}^{n} P\left(X_{i} e^{-r \tau_{i}}>x, N(t)=n\right) \\
= & I_{1}-I_{2} .
\end{aligned}
$$

By (4.19) and (4.20) of Li et al. [23], we obtain for each $t>0$,

$$
\begin{gathered}
\lim _{x \rightarrow \infty} \frac{I_{1}}{\int_{0^{-}}^{t} \bar{F}\left(\mathrm{xe}^{\mathrm{rs}}\right) \tilde{\lambda}(\mathrm{d} s)}=1, \\
\lim _{m \rightarrow \infty} \lim _{x \rightarrow \infty} \frac{I_{2}}{\int_{0^{-}}^{t} \bar{F}\left(\mathrm{xe}^{\mathrm{rs}}\right) \tilde{\lambda}(\mathrm{d} s)}=0 .
\end{gathered}
$$

By (40)-(42), we obtain for each $t>0$,

$$
\psi(x, t) \gtrsim \int_{0^{-}}^{t} \bar{F}\left(\mathrm{xe}^{\mathrm{rs}}\right) \tilde{\lambda}(\mathrm{d} s) .
$$

For each $t>0$ and $x>0$, Lemma 5 implies

$$
\begin{aligned}
\psi(x, t) & \leq P\left(\sum_{i=1}^{N(t)} X_{i} e^{-r \tau_{i}}-p_{*}(t)>x\right) \\
& =P\left(\sum_{i=1}^{N(t)} X_{i} e^{-r \tau_{i}}+p^{*}(t)>x\right) \\
& =P\left(D(t)+p^{*}(t)>x\right) .
\end{aligned}
$$

By Lemma 1 (2), we know that the distribution of $D(t)$ belongs to the class $L \cap D$. Hence, by Lemmas 3 and 4 , it holds that

$$
P\left(p^{*}(t)>x\right)=o(P(D(t)>x)) .
$$

Hence, by (44), Lemmas 2 (1) and 4, we obtain

$$
\begin{aligned}
\psi(x, t) \leqslant P & (D(t)>x) \\
& \sim \int_{0^{-}}^{t} \bar{F}\left(\mathrm{xe}^{\mathrm{rs}}\right) \tilde{\lambda}(\mathrm{d} s) .
\end{aligned}
$$




\section{Data Availability}

No data were used to support this study.

\section{Conflicts of Interest}

The authors declare that there are no conflicts of interest regarding the publication of this paper.

\section{Acknowledgments}

This work was supported by the National Natural Science Foundation of China (no. 11401418), the 333 Talent Training Project of Jiangsu Province, and the Jiangsu Province Key Discipline in the 13th Five-Year Plan.

\section{References}

[1] S. Asmussen, "Subexponential asymptotics for stochastic processes: extremal behavior, stationary distributions and first passage probabilities," The Annals of Applied Probability, vol. 8, no. 2, pp. 354-374, 1998.

[2] Y. Chen and K. W. Ng, "The ruin probability of the renewal model with constant interest force and negatively dependent heavy-tailed claims," Insurance: Mathematics and Economics, vol. 40, no. 3, pp. 415-423, 2007.

[3] X. Hao and Q. Tang, "A uniform asymptotic estimate for discounted aggregate claims with subexponential tails," Insurance: Mathematics and Economics, vol. 43, no. 1, pp. 116-120, 2008.

[4] V. Kalashnikov and D. Konstantinides, "Ruin under interest force and subexponential claims: a simple treatment," Insurance: Mathematics and Economics, vol. 27, no. 1, pp. 145-149, 2000.

[5] C. Klüppelberg and U. Stadtmüller, "Ruin probabilities in the presence of heavy-tails and interest rates," Scandinavian Actuarial Journal, vol. 1998, no. 1, pp. 49-58, 1998.

[6] D. Konstantinides, Q. Tang, and G. Tsitsiashvili, "Estimates for the ruin probability in the classical risk model with constant interest force in the presence of heavy tails," Insurance: Mathematics and Economics, vol. 31, no. 3, pp. 447-460, 2002.

[7] K. Wang, Y. Wang, and Q. Gao, "Uniform asymptotics for the finite-time ruin probability of a dependent risk model with a constant interest rate," Methodology and Computing in Applied Probability, vol. 15, no. 1, pp. 109-124, 2013.

[8] Y. Dong, K. C. Yues, and G. Wang, "Pricing credit derivatives under a correlated regime-switching hazard processes model," Journal of Industrial \& Management Optimization, vol. 13, no. 3, pp. 1395-1415, 2017.

[9] Q. Fu, P.-P. Gu, and J.-R. Wu, "Decentralized iterative learning control for large-scale interconnected linear systems with fixed initial shifts," International Journal of Control, Automation and Systems, vol. 15, no. 5, pp. 1991-2000, 2017.

[10] J. Li, "A note on the finite-time ruin probability of a renewal risk model with Brownian perturbation," Statistics \& Probability Letters, vol. 127, pp. 49-55, 2017.

[11] X. Peng, W. Su, and Z. Zhang, "On a perturbed compound poisson risk model under a periodic threshold-type dividend strategy," Journal of Industrial \& Management Optimization, vol. 13, no. 5, pp. 1-20, 2017.

[12] J. Peng and D. Wang, "Asymptotics for ruin probabilities of a non-standard renewal risk model with dependence structures and exponential Lévy process investment returns," Journal of Industrial \& Management Optimization, vol. 13, no. 1, pp. 155-185, 2017.

[13] J. Peng and D. Wang, "Uniform asymptotics for ruin probabilities in a dependent renewal risk model with stochastic return on investments," Stochastics: An International Journal of Probability and Stochastic Processes, vol. 90, no. 3, pp. 432-471, 2018.

[14] Y. Yang, K. Wang, J. Liu et al., "Asymptotics for a bidimensional risk model with two geometric Lévy price processes," Journal of Industrial \& Management Optimization, vol. 15, no. 2, pp. 481-505, 2019.

[15] W. Yu, Y. Yong, G. Guan, Y. Huang, W. Su, and C. Cui, "Valuing guaranteed minimum death benefits by cosine series expansion," Mathematics, vol. 7, no. 9, p. 835, 2019.

[16] Z. Zhang, Y. Yong, and W. Yu, "Valuing equity-linked death benefits in general exponential Lévy models," Journal of Computational and Applied Mathematics, vol. 365, Article ID 112377, 2020.

[17] H. Albrecher and J. L. Teugels, "Exponential behavior in the presence of dependence in risk theory," Journal of Applied Probability, vol. 43, no. 1, pp. 257-273, 2006.

[18] M. Boudreault, H. Cossette, D. Landriault, and E. Marceau, "On a risk model with dependence between interclaim arrivals and claim sizes," Scandinavian Actuarial Journal, vol. 2006, no. 5, pp. 265-285, 2006.

[19] H. Cossette, E. Marceau, and F. Marri, "On the compound poisson risk model with dependence based on a generalized Farlie-Gumbel-Morgenstern copula," Insurance: Mathematics and Economics, vol. 43, no. 3, pp. 444-455, 2008.

[20] A. L. Badescu, E. C. K. Cheung, and D. Landriault, "Dependent risk models with bivariate phase-type distributions," Journal of Applied Probability, vol. 46, no. 1, pp. 113-131, 2009.

[21] S. Asmussen, H. Schmidli, and V. Schmidt, "Tail probabilities for non-standard risk and queueing processes with subexponential jumps," Advances in Applied Probability, vol. 31, no. 2, pp. 422-447, 1999.

[22] A. V. Asimit and A. L. Badescu, "Extremes on the discounted aggregate claims in a time dependent risk model," Scandinavian Actuarial Journal, vol. 2010, no. 2, pp. 93-104, 2010.

[23] J. Li, Q. Tang, and R. Wu, "Subexponential tails of discounted aggregate claims in a time-dependent renewal risk model," Advances in Applied Probability, vol. 42, no. 04, pp. 11261146, 2010.

[24] K. Wang, L. Chen, Y. Yang, and M. Gao, “The finite-time ruin probability of a risk model with stochastic return and brownian perturbation," Japan Journal of Industrial and Applied Mathematics, vol. 35, no. 3, pp. 1173-1189, 2018.

[25] P. Embrechts, C. Klüppelberg, and T. Mikosch, Modelling Extremal Events for Insurance and Finance, Springer, Berlin, Germany, 1997.

[26] P. Embrechts, C. M. Goldie, and N. 1. Veraverbeke, "Subexponentiality and infinite divisibility," Zeitschrift für Wahrscheinlichkeitstheorie und Verwandte Gebiete, vol. 49, no. 3, pp. 335-347, 1979.

[27] V. I. Piterbarg, Asymptotic Methods in the Theory of Gaussian Processes and Fields, American Mathematical Society, Providence, RI, USA, 1996. 